Artículo Especial

\title{
El aborto en Chile: aspectos epidemiológicos, históricos y legales
}

\author{
Enrique Donoso S. ${ }^{1}$, Claudio Vera P-G. ${ }^{1,2}$ \\ ${ }^{1}$ División de Obstetricia y Ginecología, Unidad de Medicina Materno-Fetal, ${ }^{2}$ Unidad de Medicina Basada en la Evidencia. \\ Escuela de Medicina, Pontificia Universidad Católica de Chile.
}

\section{RESUMEN}

Antecedentes: En Chile, el aborto es ilegal en cualquier circunstancia. Objetivos: Describir la evolución epidemiológica del aborto en Chile, su relación con la introducción de los métodos anticonceptivos (MAC) y los cambios históricos de su legalidad. Adicionalmente, revisar los aspectos metodológicos de las estimaciones del aborto ilegal en Chile. Resultados: La tasa de mortalidad materna por aborto se ha reducido drásticamente en Chile, desde una de las más altas en Latinoamérica a principios del siglo XX a una de las más bajas en la actualidad. La importante reducción observada después de la introducción de los MAC refleja los cambios culturales y avances en el desarrollo económico y sanitario del país. El cambio de status del aborto legal a ilegal no se asoció a un aumento de las muertes maternas relacionadas a aborto que mantienen una reducción sostenida. Se desconoce con certeza la magnitud del aborto ilegal en Chile, metodologías utilizadas para su estimación podrían sobrestimarlo ampliamente si no se consideran factores condicionantes. Conclusión: Chile ha logrado descender drásticamente la mortalidad materna por aborto sin recurrir a la legalización o su despenalización, respetando al embrión humano desde la concepción. Se requiere considerar una metodología apropiada para el monitoreo de la prevalencia y causas de aborto ilegal que permita focalizar las intervenciones preventivas en la población vulnerable.

\section{PALABRAS CLAVES: Aborto, mortalidad materna, anticoncepción, planificación familiar, Chile}

\section{SUMMARY}

Background: In Chile abortion is illegal in any circumstance. Objectives: To describe epidemiological evolution of abortion in Chile, its relationship with the introduction of contraceptive methods (CM) and historical changes on its legal status. In addition, we review methodological issues of estimates of illegal abortion in Chile. Results: Maternal mortality rate to abortion have been reduced drastically in Chile, from one of the highest in Latin America at the beginning of the XX century to one of the lowest rates currently. The important reduction observed after the introduction of $\mathrm{CM}$ reflects cultural changes as well as economical and sanitary progress of the country. Legal status change from legal to illegal was not related to an increase in maternal mortality rate due to abortion that maintains a sustained reduction. Magnitude of illegal abortion in Chile is unknown; methodologies used for estimation may largely overestimate prevalence if conditionings factors are not taken into account. Conclusion: Chile has managed to drastically reduce maternal mortality due to abortion without recourse to legalization or decriminalization, respecting the human embryo from conception. A proper methodology is required to monitoring prevalence and causes of illegal abortion in order to focusing preventive interventions in the vulnerable population.

KEY WORDS: Abortion, maternal mortality, contraception, family planning, Chile 


\section{INTRODUCCIÓN}

Se define aborto a la interrupción espontánea o inducida del embarazo antes de la viabilidad fetal $(1,2)$. El momento en el cual es posible la sobrevida extrauterina no tiene un definición uniforme, la $10^{\mathrm{a}}$ Revisión de la Clasificación Internacional de Enfermedades (CIE 10) recomienda que para el reporte de estadísticas vitales se considere muerte fetal o nacido vivo con peso al nacer de $500 \mathrm{~g}$ o más y se considere la edad gestacional de 22 semanas o una talla de $25 \mathrm{~cm}$ o más cuando el peso no esté disponible (3). Desde el punto de vista clínico, se reconoce un periodo "periviable" entre las $20+0$ y $25+6$ semanas donde la sobrevida es creciente a partir de las 22 semanas y depende tanto de la condición fetal como del progreso en el manejo de la prematurez extrema (4). La frecuencia del aborto espontáneo es difícil de precisar. Se estima que aproximadamente el $15 \%$ de los embarazos clínicos terminan en aborto espontáneo, sin embargo, si se consideran los embarazos subclínicos la frecuencia de aborto espontáneo oscila entre el 30$50 \%$ de las gestaciones (5).

\section{TIPOS DE ABORTO}

Dentro de las diferentes clasificaciones de aborto se reconocen 2 grandes causas: i. Aborto espontáneo y, ii. Aborto inducido. Como su nombre lo indica se trata de la forma como se produce el aborto. El primero se refiere a la interrupción natural del embarazo, sin mediar ningún procedimiento para su desencadenamiento, y que habitualmente es el producto de alguna alteración genética que lleva a la pérdida precoz de la gestación $(6,7)$. El segundo se refiere a la interrupción artificial de la gestación mediante procedimientos mecánicos (dilatación cervical y legrado uterino; dilatación y aspiración vacum, etc.), o uso de drogas (mifepristona o RU 486, metotrexato, misoprostol, etc.) (8). En países donde el aborto inducido es legal, y justificado para interrumpir la gestación frente a patologías maternas diversas que potencialmente pueden agravarse en el transcurso del embarazo, o previo a tratar una patología materna severa, se denomina aborto médico. También se utiliza para interrumpir la gestación cuando el feto tiene alguna anomalía estructural compatible o supuestamente incompatible con la vida intra o extrauterina, o por problemas de tipo genético compatible (trisomía 21) o supuestamente incompatible (trisomías 13 y 18) con la vida extrauterina, este tipo de aborto se denomina aborto eugenésico. Dentro del aborto inducido legal también se reconocen variadas indicaciones como: por libre demanda o por violación/incesto, que tienen en común una madre y feto sin patologías evidenciables.

\section{ABORTO SEGURO/INSEGURO}

La frecuencia de aborto inducido es difícil de precisar, especialmente en aquellos países donde hay restricción legal del aborto, mientras que en aquellos países donde el aborto inducido es legal las cifras son más reales. Es así que en EEUU en 2008 se realizaron 1.212 .000 abortos legales, con una tasa de aborto inducido de 19,4/1000 mujeres de 15-44 años (9), en estas circunstancias, la muerte de la mujer como consecuencia del procedimiento es excepcional porque es realizado por médicos, en ambiente aséptico y anestesia cuando es necesario; el aborto inducido en estas condiciones se denomina aborto seguro.

En EEUU, en el periodo 1988-1997, la tasa de letalidad por aborto inducido fue de 0,7 muertes/100.000 abortos legales, aumentando exponencialmente en $38 \%$ por cada semana de edad gestacional adicional, con un riesgo relativo de mortalidad relacionada al aborto de 76,6 (IC 95\% 32,5 a 180,8 ) en embarazos $\geq 21$ semanas (10). En el período 1998-2010, no cambió la tasa de mortalidad relacionada al aborto $(0,7$ muertes $/ 100.000$ procedimientos), aumentando con la edad gestacional $(0,3 / 100.000$ en gestaciones $\leq 8$ semanas y de $6,7 / 100.000$ en gestaciones $\geq 18$ semanas) (11).

En países y regiones con menor desarrollo, baja cobertura de métodos de planificación familiar y asistencia médica, aumenta el riesgo de que una mujer recurra al aborto inducido, practicado por personal no calificado y con procedimientos inapropiados e insalubres, situación definida como aborto inseguro por la alta frecuencia de mortalidad y morbilidad materna, generando un grave problema de salud pública para esos países, como también un grave problema para la familia por la madre ausente, muchas veces principal proveedora del grupo familiar.

La Organización Mundial de la Salud (OMS), estima que aproximadamente 20 millones de embarazos terminan anualmente en aborto inseguro, la gran mayoría de ellos en países en desarrollo y responsables de 80.000 muertes anuales ( 400 muertes maternas/100.000 abortos) y recomienda a los países tomar las medidas para transformar el aborto inseguro en aborto seguro (12). En los países en desarrollo el aborto inseguro es una de las principales causas de muerte materna por infección, hemorragia o intoxicación por sustancias utilizadas para inducir el aborto. De ahí que la OMS recomienda a los países transformar el aborto inseguro en seguro mediante su legalización, facilitando el acceso al aborto seguro y a los métodos modernos de anticoncepción, como también la capacitación de los proveedores para el adecuado tratamiento de las complicaciones del aborto inseguro (8). 


\section{EVOLUCIÓN HISTÓRICA DE LA LEGALIDAD DEL ABORTO EN CHILE (13)}

El Código Penal de Chile de 1874 consideró el aborto inducido como un delito. En 1931, con la creación del Código Sanitario, mediante el Decreto con Fuerza de Ley $\mathrm{N}^{\circ} 226$, se estableció que "Por razones terapéuticas, se podía interrumpir un embarazo o practicar una intervención para hacer estéril a una mujer, procedimientos que requieren de la opinión documentada de tres facultativos" (Art. 226). En 1968, se deroga el Código Sanitario de 1931, y entra en vigencia un nuevo Código Sanitario (Decreto con Fuerza de Ley № 725). Ese decreto estableció que "Sólo con fines terapéuticos se podrá interrumpir un embarazo. Para proceder esta intervención se requerirá la opinión documentada de dos médicos-cirujanos" (artículo 119). En 1989, el gobierno Militar modificó el Código Sanitario, y cambia el artículo 119 estableciendo: "No podrá ejecutarse ninguna acción cuyo fin sea provocar un aborto", y por sobre esta normativa, la legislación chilena establece en el artículo 19 de su Constitución Política de 1980 que: "La ley protege la vida del que está por nacer".

El 31 de enero de 2015, la Presidenta de la República de Chile, Dra. Michelle Bachelet Jeria, envía mensaje con el que inicia un proyecto de ley (Mensaje $\left.\mathrm{N}^{\circ} 1230-362\right)$, que regula la despenalización de la interrupción voluntaria del embarazo en tres causales (14): 1. Peligro de la vida de la mujer embarazada. 2. Embrión o feto que padezca una alteración estructural congénita o genética incompatible con la vida extrauterina. 3. Embarazo producto de una violación. Proyecto de ley aprobado el 17 de marzo de 2016 en su primer trámite constitucional por la Cámara de Diputados y actualmente desde el 26 de octubre de 2016 en su segundo trámite constitucional en el Senado con urgencia simple.

\section{TRANSICIÓN DEMOGRÁFICA DE CHILE: EFECTO DE LA PLANIFICACIÓN FAMILIAR}

Chile en la actualidad se encuentra cursando la denominada transición demográfica avanzada. Esto significa que el país tiene una baja natalidad y mortalidad, lo que determina un crecimiento natural bajo, propio de los países desarrollados o cercanos al desarrollo. Chile en 2013 presentó una tasa global de fecundidad de 1,79 hijos-hijas/1000 mujeres 15-49 años (15), inferior a la tasa de recambio poblacional definida como de 2,1 hijo-hija por mujer; esto significa que en la actualidad los hijos nacidos por cada mujer no serían suficientes para renovar la población al momento del fallecimiento de sus progenitores.

En la Figura 1, se presenta la transición demográfica de Chile entre los años 1900 y 2004 (16). Observamos que en 1900 el país tenía una alta tasa de natalidad y mortalidad con un crecimiento natural inferior al 0,5\% anual. Con el desarrollo del país, la mortalidad general desciende pero mantiene una alta natalidad que trae como consecuencia un crecimiento natural en ascenso cercano al 2,5\% anual a mediados de la década de los 60 del siglo $X X$. En esa década se introduce en Chile los métodos anticonceptivos con la consiguiente baja de la natalidad, que asociada al continuo descenso de la mortalidad produce un importante descenso del crecimiento natural que en la actualidad es inferior al $1 \%$ anual. Estos cambios producen en la población una asimetría en sus diferentes grupos etarios, siendo los más destacables el envejecimiento poblacional por un aumento de la población de 60 o más años y una disminución de la población de 15 o menos años.

\section{EPIDEMIOLOGÍA DEL ABORTO EN CHILE}

Los métodos anticonceptivos se introdujeron en Chile en la década de los 60 del siglo recién pasado, posterior a lo cual se observa una progresiva reducción de la tasa global de fecundidad de 5 hijoshijas por mujer en 1960 a 1,79 hijos-hijas por mujer en el 2013. Esto ha permitido a la mujer alcanzar un mayor desarrollo personal en lo educacional y económico, como también un mejor bienestar de sus hijos en lo que se refiere a educación y vivienda especialmente.

La posibilidad de controlar el número de embarazos permitió a la mujer no recurrir al aborto como método de control de fecundidad, y reducir el alto costo familiar y del país que implican las complicaciones derivadas del aborto inducido. En la Figura 2 se presenta la tendencia ascendente de la relación entre el número de abortos/1000 nacimientos entre los años 1937 y 1964 (17).

Muñoz y cols (18), en 1980 presentaron un análisis de 30 años (1950-1979) de la mortalidad materna en el hospital Guillermo Grant Benavente de Concepción ( $8^{a}$ Región, Chile). En el período analizado, hubo 527 muertes maternas y 175.152 nacidos vivos, con una razón de mortalidad materna $300,9 / 100.000$ nacidos vivos. De ellas, 213 muertes $(40,4 \%)$ fueron relacionadas al aborto, con una mortalidad materna por aborto de 121,6/100.000 nacidos vivos; 187 muertes por infección, de las cuales 57 casos $(30,5 \%)$ correspondieron a septicemia por clostridium perfringens.

Armijo y Monreal (19), caracterizaron la epidemiología del aborto en Chile, antes de la introducción de los métodos anticonceptivos. Como se aprecia en la Tabla I, en 1963 la mortalidad materna en Chile por complicaciones del aborto correspondía al $38,8 \%$ del total de muertes maternas (312 muertes por aborto), con una mortalidad materna global de 273/100.000 nacidos vivos, una de las más altas de Latinoamérica. En ese estudio se verificó 


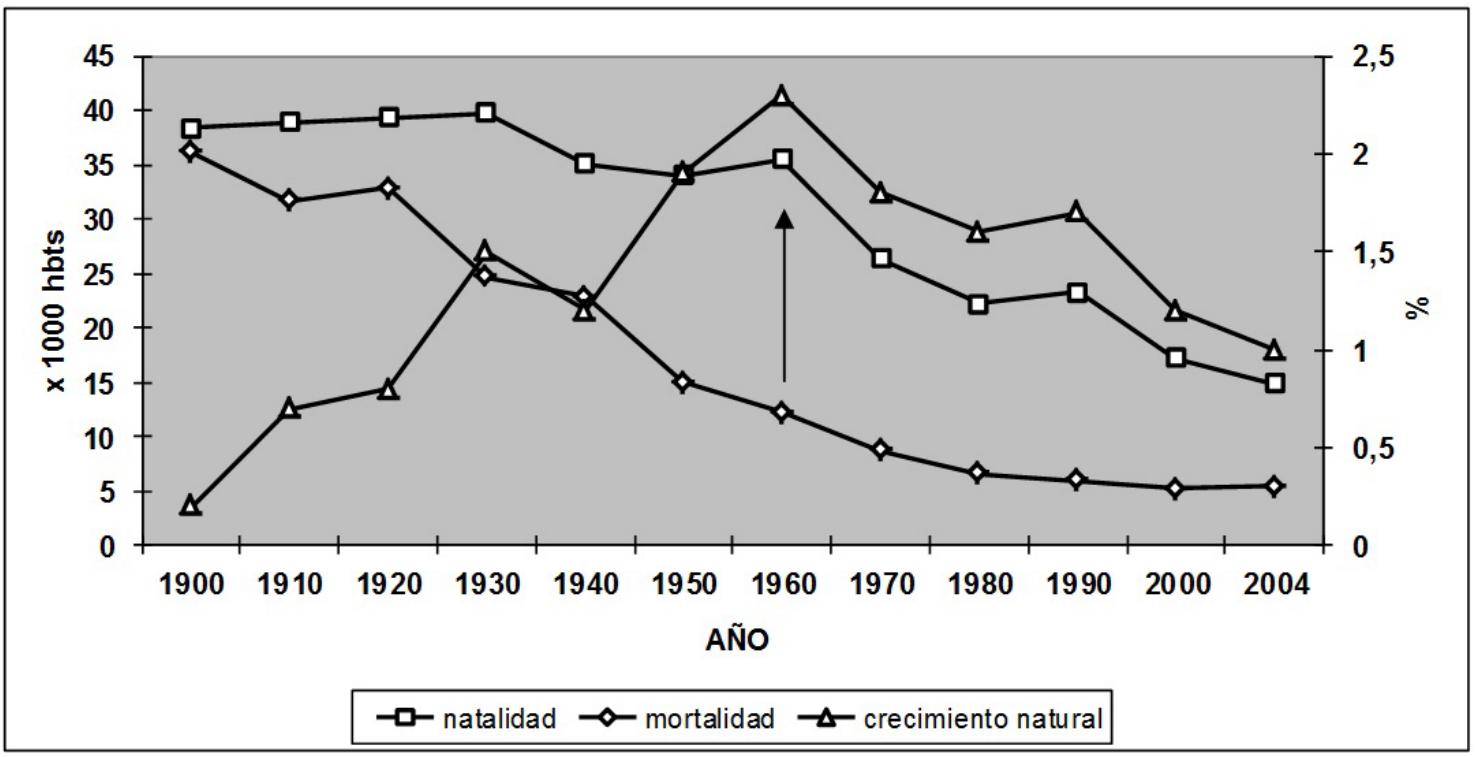

Figura 1. Transición demográfica, Chile 1900-2004 (flecha indica la introducción de los métodos anticonceptivos) (16).

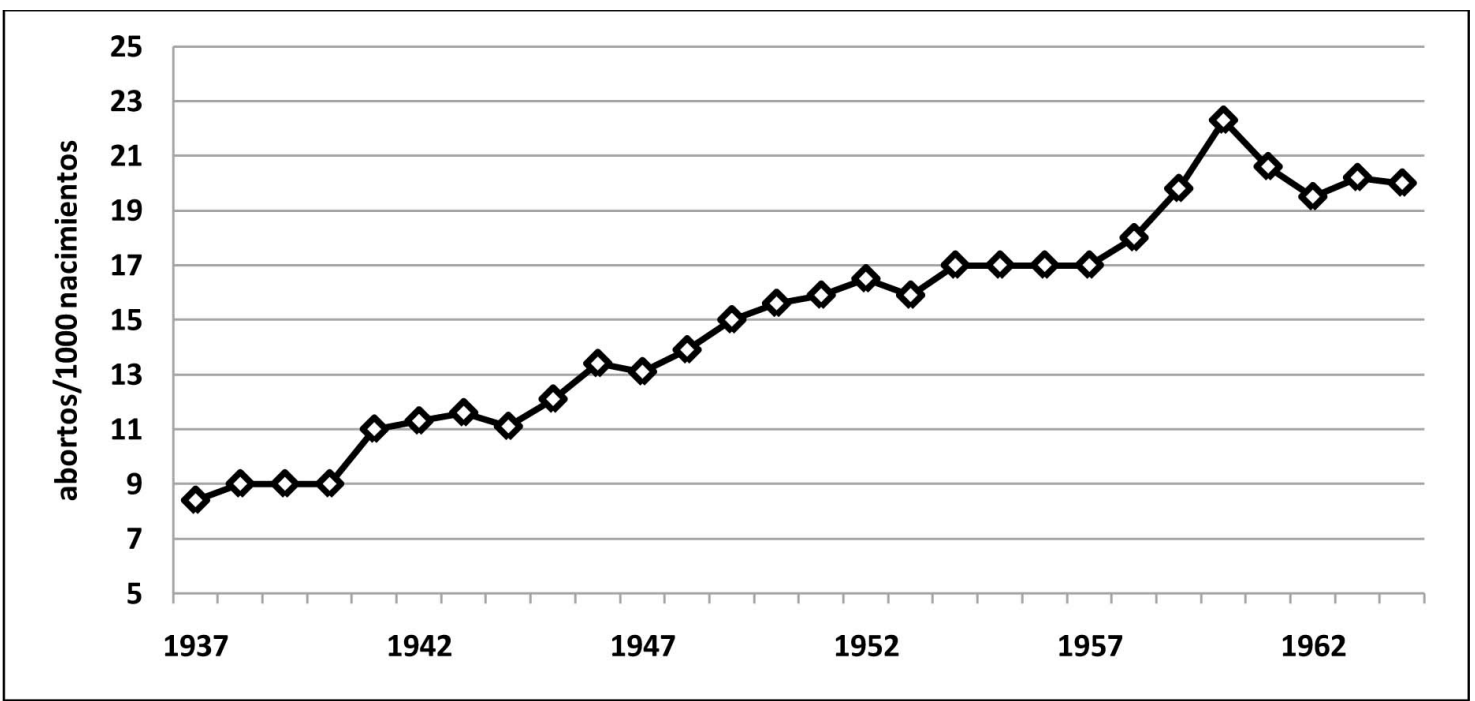

Figura 2. Tendencia de la relación entre el número de abortos/1000 nacimientos, Chile 1937-1964. Realizada con los datos publicados por Armijo y Requena (17).

que las mujeres que recurrieron al aborto, el $43,7 \%$ tenía antecedentes de 1 a 3 abortos inducidos previos y el $15,5 \%$ tenía 10 o más; el 34\% tenía entre $25-29$ años y el $66 \%$ tenía entre 1 y 3 hijos vivos, estos antecedentes sugerían que la mujer recurría al aborto como un método de planificación familiar. Además para provocar el aborto, el $45,1 \%$ utilizaba sonda intrauterina, el $35,6 \%$ raspado uterino, el $8,1 \%$ lavado intrauterino, y el $11,2 \%$ otros métodos. 
Tabla I

ASPECTOS DEMOGRÁFICOS DE LA MORTALIDAD MATERNA POR ABORTO, CHILE 1963 (19)

\begin{tabular}{ll}
\hline Variable & \multicolumn{1}{c}{$\mathrm{N}^{\circ}$ de casos y tasas } \\
\hline Nacidos vivos & 294.175 \\
Muertes maternas & 803 \\
Mortalidad materna & $273 / 100.000$ nacidos vivos \\
$\begin{array}{l}\text { Muertes maternas por } \\
\text { aborto }\end{array}$ & 312 \\
$\begin{array}{l}\text { Mortalidad materna por } \\
\text { aborto }\end{array}$ & $106 / 100.000$ nacidos vivos \\
$\begin{array}{l}\text { Porcentaje de mortalidad } \\
\text { materna por aborto }\end{array}$ & $38,8 \%$ \\
\hline
\end{tabular}

Los métodos utilizados explica la alta mortalidad y morbilidad en las mujeres que recurrían al aborto, y el principal motivo eran problemas económicos en el $48 \%$ de los casos.

\section{EFECTO DE LA PLANIFICACIÓN FAMILIAR SOBRE LA MORTALIDAD MATERNA POR ABORTO}

A mediados de la década de los 60 el Ministerio de Salud crea el programa de Regulación de la Fertilidad, introduciendo en Chile los métodos de planificación familiar, siendo uno de sus principales objetivos reducir el aborto inseguro y como consecuencia la muerte de la mujer. Durante la década de los 70 y 80 , junto con la ampliación de la cobertura de los programas de planificación familiar, del control prenatal y la atención profesional del parto, se logró disminuir la natalidad y la muerte materna por aborto, como también las por causas directas, especialmente. En la Figura 3 se aprecia que la mortalidad materna por aborto asciende desde 1952 hasta los inicios de la década de los 60, luego se mantiene alta y estable e inicia un sostenido descenso desde 1965, y coincidente con la introducción de los métodos de planificación familiar (20).

En la Figura 4 se presenta la tendencia de la mortalidad materna por aborto entre los años 1985 y 2000 , excluye las muertes por embarazo ectópico, enfermedad del trofoblasto y otros productos anómalos de la gestación; se aprecia un evidente descenso desde $11,7 / 100.000$ nacidos vivos en 1985 a 4,8/100.000 nacidos vivos en el 2000 (21). En 1985 , el $24 \%$ de las muertes se produjo por aborto ilegal y el $17 \%$ por complicaciones del aborto; es posible que estas últimas fueran atribuibles en una proporción importante al aborto inseguro. En el 2000, las causas de las muertes por aborto fueron: $8 \%$ aborto espontáneo, $8 \%$ aborto médico, $8 \%$ otro tipo de aborto y $76 \%$ por aborto no especificado. En ese estudio, en el año 2000, el promedio de edad de las mujeres fallecidas por aborto fue de $20,9 \pm 6,6$ años, el $41,7 \%$ eran solteras y el $58,3 \%$ solo tenía educación básica (21).

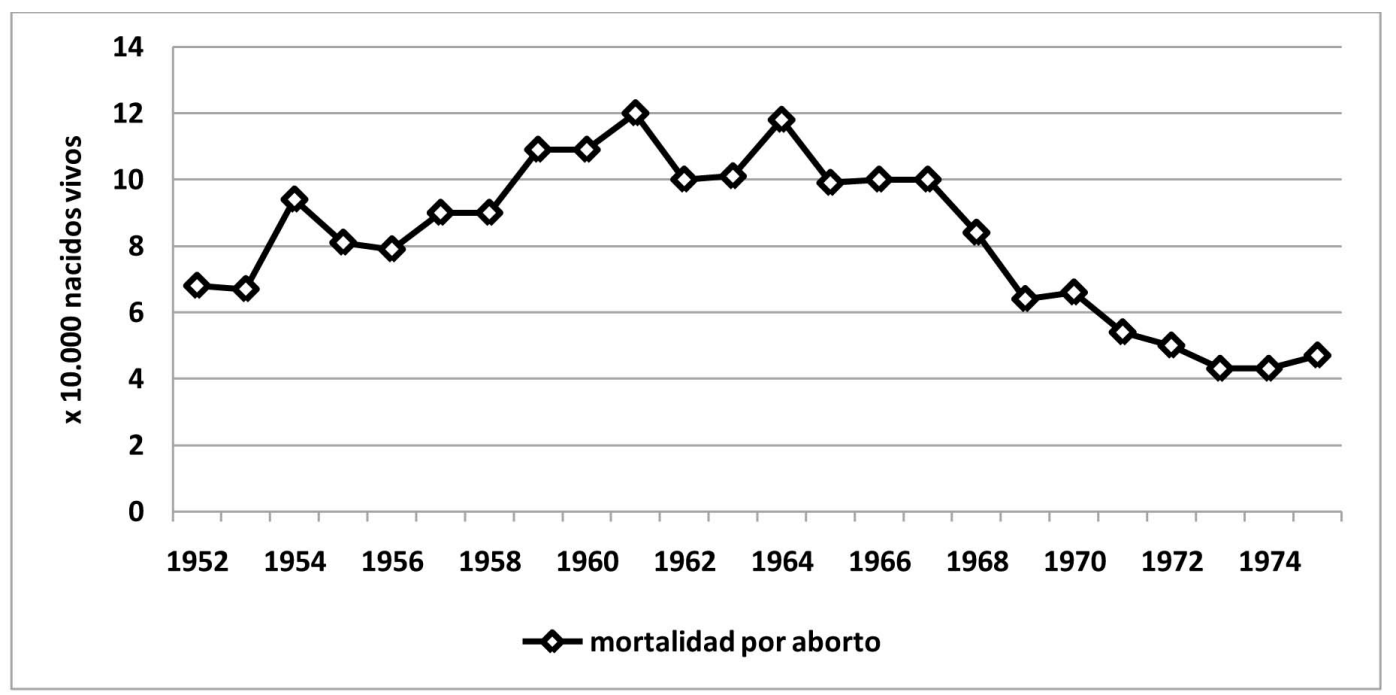

Figura 3. Mortalidad materna por aborto (x 10.000 nacidos vivos), Chile 1952-1975. Realizada con los datos publicados por Molina y cols (20). 


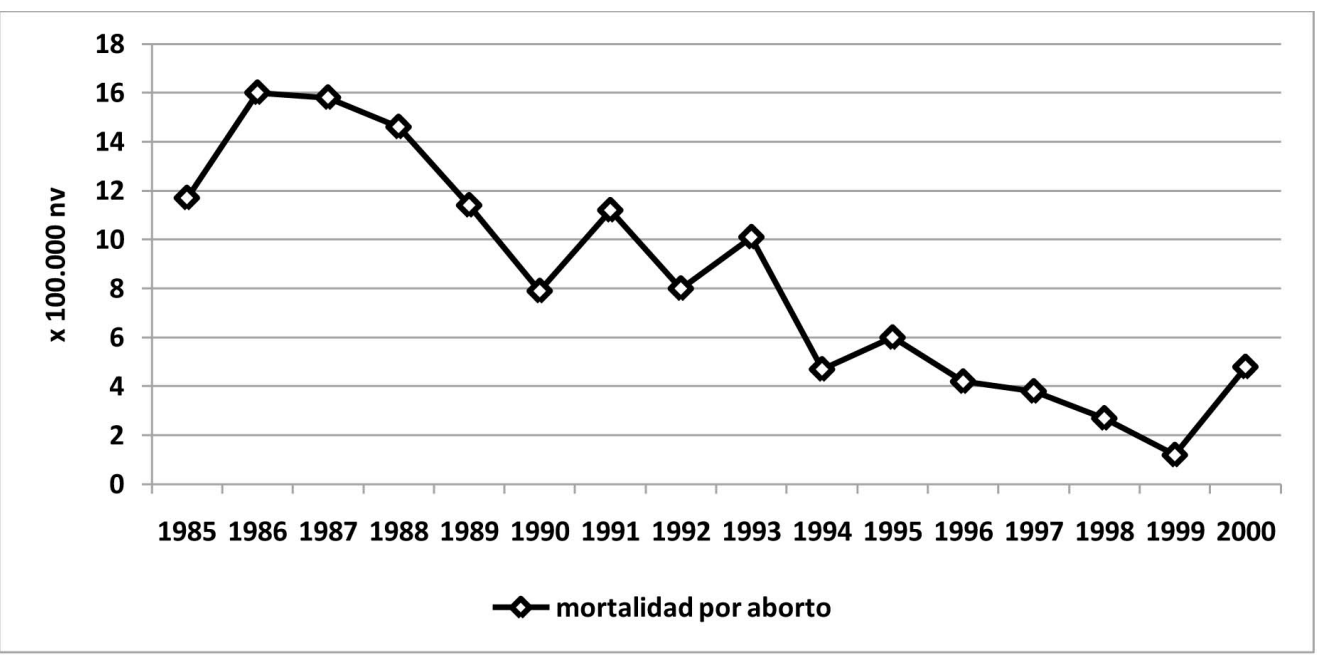

Figura 4. Mortalidad materna por aborto (x 100.000 nacidos vivos), Chile 1985-2000. Realizada con los datos publicados por Szot y Moreno (21).

En la Figura 5 se presenta la tendencia de la mortalidad materna por aborto (x 100.000 nacidos vivos) en el período 1990-2011. La tendencia presenta un descenso significativo en el periodo $(r=$ $-0,8342 ; p<0,001)(22)$. Esta tendencia el Ministerio de Salud lo atribuye al amplio acceso y gratuito a los métodos de planificación familiar, en la atención pública primaria de salud (23).
En el período 2000-2009 hubo un total de 38 muertes por aborto (códigos CIE 10: O03-O06) correspondientes al $8,8 \%$ del total de muertes maternas del período, con una mortalidad materna por aborto de 1,6/100.000 nacidos vivos y con una tendencia significativamente descendente $(r=-0,712$; $\mathrm{p}=0,023)(24)$.

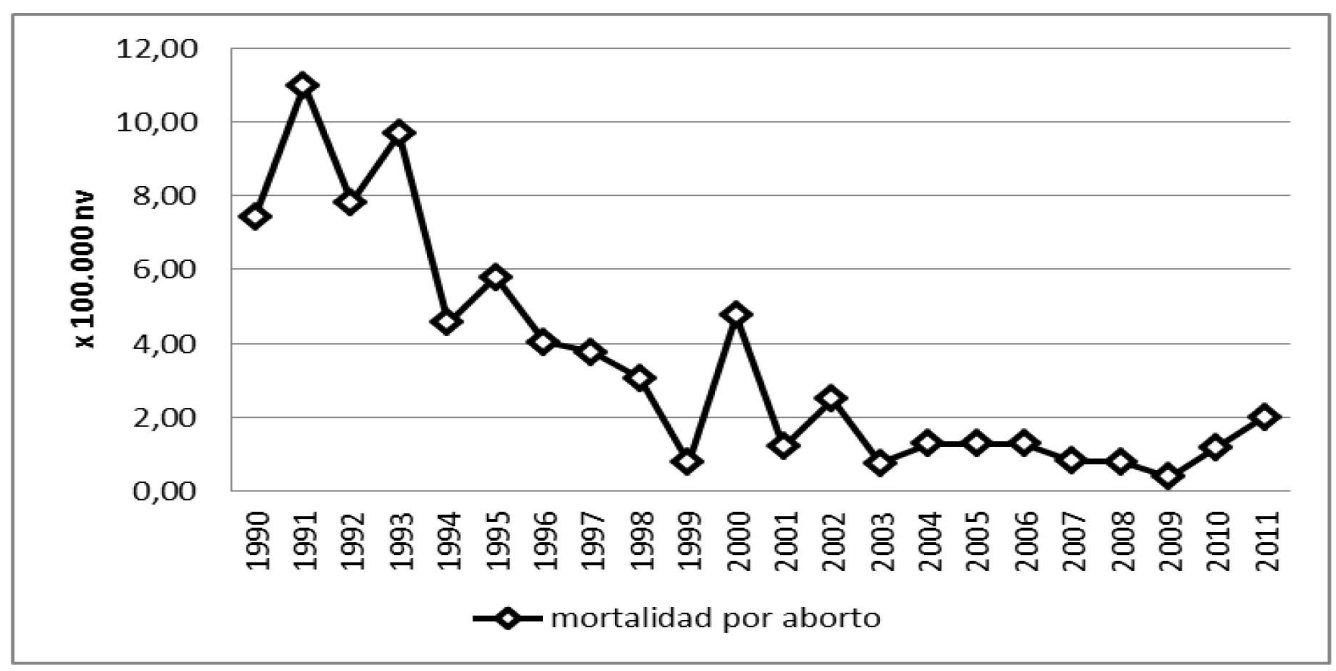

Figura 5. Tendencia de la mortalidad materna por aborto (x 100.000 nv), Chile 1990-2011. Realizada por los autores con datos de los Anuarios de Estadísticas Vitales del Instituto Nacional de Estadísticas (22). 
Podemos concluir que los métodos de planificación familiar han permitido a la mujer chilena no recurrir al aborto para planificar su familia, y han permitido reducir drásticamente la mortalidad materna asociada aborto desde $106 / 100.000$ nacidos vivos en 1963 (19) a 1,6/100.000 nacidos vivos en la actualidad (24), de ahí que en Chile la mortalidad materna por aborto reduce fuertemente su impacto como problema de salud pública.

\section{ASPECTOS EPIDEMIOLÓGICOS DE LA PLANI- FICACIÓN FAMILIAR Y LA ANTICONCEPCIÓN EN CHILE}

Como ya mencionamos los métodos de planificación familiar fueron introducidos en Chile por el Ministerio de Salud en la década de los 60 del siglo recién pasado, con el objetivo que las familias pudiesen regular su fecundidad y reducir la mortalidad materna como consecuencia del aborto inducido inseguro, objetivo plenamente cumplido, según las actuales tasas de mortalidad materna por aborto y la tendencia significativamente descendente desde la incorporación de los diferentes métodos anticonceptivos. Es así, que en la actualidad la probabilidad de morir de la mujer como consecuencia del aborto es de 0,046/100.000 mujeres de 15-49 años (1 en 2.000.000 mujeres de 15-49 años) (25).

En la Encuesta de Calidad de Vida y Salud, realizadas en el 2000, la información nacional sobre uso de métodos anticonceptivos se presenta resumida en Tablas II y III (26). De la información obtenida en esa encuesta, podemos ver que, pese a que han transcurrido 16 años, la población encuestada y representativa a nivel nacional, mostró una importante aceptación del uso de alguna forma de anticoncepción, alcanzando casi al $50 \%$ de la población encuestada mayor de 15 años, que los métodos hormonales y los dispositivos intrauterinos $(\sim 33 \%)$ son los de mayor prevalencia de uso, de las personas que no usan métodos anticonceptivos solo el $1,9 \%$ se opone a su uso, y que el $77,1 \%$ de las/os usuarios tuvieron supervisión profesional. Es muy posible que en el tiempo transcurrido la prevalencia de usuarias de métodos anticonceptivos haya aumentado, lo que se refleja en una baja natalidad y mortalidad materna sin cambios significativos desde el 2000 en adelante (24).

\section{FECUNDIDAD, MUERTE POR ABORTO Y ANTICONCEPCIÓN EN LA ADOLESCENCIA}

Es importante analizar la contribución de las madres adolescentes a la natalidad del país. Como se aprecia en la Tabla IV, el 15,9\% de los nacidos vivos en el período 2000-2009 correspondió a adolescentes (10-14 años el 0,4\% y de 15-19 años el $15,5 \%$ del total de nacidos vivos), sin cambios en la tendencia tanto en adolescentes <15 años como en las entre 15-19 años, y con una mortalidad materna general de 9,8/100.000 nacidos vivos en adolescentes menores de 15 años y de 11,3/100.000 nacidos vivos en adolescentes de 15-19 años, siendo estas tasas las más baja de todos los grupos etarios. En el periodo no se registró muertes por aborto en menores de 15 años y la mortalidad por aborto en adolescentes de 15-19 años fue de $1,61 / 100.000$ nacidos vivos, sin embargo, la mortalidad materna en adolescentes representa el 10\% del total de las muertes maternas del período (24).

Tabla II

TIPO DE MÉTODOS ANTICONCEPTIVOS EN LA POBLACIÓN NACIONAL, CHILE 2000 (26)

\begin{tabular}{lcc}
\hline Métodos & Frecuencia & $\%$ \\
\hline No usa & 5.897 .511 & 52,5 \\
Usa píldoras & 1.747 .238 & 15,6 \\
Tiene dispositivo intrauterino & 1.879 .190 & 16,7 \\
Usa inyecciones & 57.406 & 0,5 \\
Usa condón & 517.830 & 4,6 \\
Evita los días fértiles & 144.423 & 1,3 \\
Usa diafragma, espuma, gel & 11.175 & 0,1 \\
Se practicó esterilización femenina & 854.001 & 7,6 \\
Se practicó esterilización masculina & 4.730 & 0,0 \\
Realiza coito interrumpido & 32.812 & 0,3 \\
Mantiene lactancia prolongada & 17.576 & 0,2 \\
Otro & 65.863 & 0,6 \\
\hline Total & 11.252 .477 & 100,0 \\
\hline
\end{tabular}




\section{Tabla III \\ MOTIVOS POR QUÉ NO USAN MÉTODOS ANTICONCEPTIVOS, CHILE 2000 (26)}

\begin{tabular}{lcc}
\hline Motivos & Frecuencia & $\%$ \\
\hline No lo necesita & 4.914 .540 & 83,8 \\
Está embarazada & 216.713 & 3,7 \\
Quiere quedar embarazada & 128.278 & 2,2 \\
Se opone al uso de anticonceptivos & 109.472 & 1,9 \\
Por razones de salud & 176.325 & 3,0 \\
No conoce los métodos & 30.691 & 0,5 \\
Por su precio, no puede comprarlos & 16.860 & 0,3 \\
Otra & 269.453 & 4,6 \\
\hline Total & 5.897 .511 & 100,0 \\
\hline
\end{tabular}

Tabla IV

TENDENCIA DE LA NATALIDAD SEGÚN GRUPOS ETARIOS, CHILE 2000 - 2009 (24)

\begin{tabular}{lccc}
\hline $\begin{array}{l}\text { Edad } \\
\text { (años) }\end{array}$ & $\begin{array}{c}\text { Nacidos vivos } \\
\mathrm{n}(\%)\end{array}$ & $\mathrm{r}=$ & Valor $\mathrm{p}$ \\
\hline$<15$ & $10.176(0,4)$ & $-0,372$ & 0,290 * \\
$15-19$ & $371.967(15,5)$ & 0,252 & 0,482 * \\
$20-24$ & $559.289(23,3)$ & 0,410 & 0,239 * \\
$25-29$ & $576.205(24,0)$ & $-0,370$ & 0,292 * \\
$30-34$ & $501.767(20,9)$ & $-0,378$ & $0,281^{*}$ \\
$35-39$ & $296.461(12,4)$ & 0,347 & $0,327^{*}$ \\
$\geq 40$ & $84.345(3,5)$ & 0,930 & 0,001 \\
\hline
\end{tabular}

* No significativo. r: coeficiente de correlación de Pearson (2 colas).

Pese a que la natalidad y la mortalidad materna en adolescentes chilenas son una de las más bajas en Latinoamérica (27), debemos considerarlas inaceptablemente altas, ya que son evitables a través de la educación en afectividad y sexualidad, que considere tanto la postergación del inicio de la vida coital, como la prevención del embarazo, y por consecuencia del aborto inducido por embarazo no deseado o no planificado.

Según la Sexta Encuesta Nacional de la Juventud (28), el 48\% de los adolescentes entre 15 a 19 años ya ha iniciado una vida sexual activa y cada vez más precoz (hombres 16,4 años y mujeres 17,1 años), especialmente de las adolescentes mujeres que en 1997 presentaba un promedio de 18 años al inicio de su vida sexual activa. En la Séptima Encuesta Nacional de la Juventud (29) se informa que el $61 \%$ de los embarazos no planificados se produce antes de los 20 años en mujeres sexualmente activas, esta situación no es transversal según nivel socio económico, bajando a $29 \%$ en mujeres de nivel socio-económico alto. Esto habla de una mejor educación en sexualidad y mejor acceso a la anticoncepción en el estrato socio-económico alto.

En el último informe del MINSAL sobre la situación actual del embarazo adolescente en Chile (30) se aprecia un importante aumento de usuarias de anticonceptivos en adolescentes. Es así que hubo un aumento del $84,6 \%$ en adolescentes de 10 a 14 años y de $6,8 \%$ en adolescentes de 15 a 19 años entre los años 2011 y 2012; es posible que en la actualidad el acceso de adolescentes a métodos de planificación familiar haya aumentado, lo que se reflejaría en futuras estadísticas en el número de nacidos vivos, en las tasas específicas de fecundidad y en la mortalidad general y por aborto del embarazo adolescente (Tabla V). 


\section{MINSAL. USUARIAS DE MÉTODOS ANTICONCEPTIVOS SEGÚN GRUPOS ETARIOS, CHILE 2005-2012 (30)}

\begin{tabular}{ccccccc}
\hline Año & $\begin{array}{c}\text { Población } \\
\text { bajo control }\end{array}$ & $\begin{array}{c}<15 \\
\text { años }\end{array}$ & $\begin{array}{c}15-19 \\
\text { años }\end{array}$ & $\begin{array}{c}20-34 \\
\text { años }\end{array}$ & $\begin{array}{c}35-44 \\
\text { años }\end{array}$ & $\begin{array}{c}45-54 \\
\text { años }\end{array}$ \\
\hline 2005 & 1.150 .646 & 2.831 & 109.092 & 613.077 & 425.646 & - \\
2006 & 1.165 .372 & 4.158 & 124.060 & 640.631 & 396.523 & - \\
2007 & 1.161 .559 & 3.635 & 133.750 & 631.036 & 393.138 & - \\
2008 & 1.162 .707 & 4.282 & 137.518 & 625.343 & 395.564 & - \\
2009 & 1.198 .048 & 4.784 & 143.331 & 652.893 & 397.040 & - \\
2010 & 1.237 .670 & 5.453 & 153.118 & 621.578 & 329.432 & 128.089 \\
2011 & 1.257 .226 & 6.917 & 161.856 & 647.460 & 317.502 & 123.491 \\
2012 & 1.339 .444 & 12.767 & 172.850 & 708.190 & 310.865 & 134.772 \\
\hline
\end{tabular}

Podemos resumir que la conducta sexual en adolescentes ha cambiado, con un inicio de la vida coital cada vez más precoz y por lo tanto expuestas a embarazos no planificados ni deseados, que las expone al aborto inducido, como también al riesgo de morir o enfermar inherentes al proceso reproductivo. A través de la educación en el ámbito familiar, colegios y facilitando el acceso a Programas de Salud Reproductiva se podrá disminuir el embarazo adolescente, como los riesgos que este implica.

\section{MAGNITUD DEL ABORTO ILEGAL EN CHILE}

Ha sido motivo de controversias cual es la real magnitud del aborto ilegal en Chile. Sin duda que la mortalidad materna general en Chile ha disminuido dramáticamente, y muy especialmente la mortalidad materna por aborto, como consecuencia del amplio acceso a la planificación familiar y anticoncepción, por lo que también es lícito pensar que el aborto ilegal también debiese haber disminuido; hecho acaecido no solo en Chile sino que también mundialmente. Se estima que el control de la fecundidad en países en desarrollo ha permitido reducir sobre 1 millón de muertes maternas entre 1990 y 2005, por el uso de métodos contraceptivos (31).

Uno de los cambios demográficos más importantes ocurridos en Chile es la importante y significativa reducción de los nacimientos, consecuencia de la alta prevalencia de usuarias de métodos anticonceptivos y no como consecuencia del aborto ilegal. No tenemos conocimiento que algún país en el mundo haya reducido la natalidad mediante el aborto clandestino. En Chile hay aborto clandestino, pero es posible que la mujer recurra a métodos menos cruentos como es el uso de misoprostol, generando una tasa de abortos ilegales no precisada, y que requiere ser estimada. Lo anterior determina que la cantidad de abortos ilegales estimados para un país pueden estar sobre o subestimados dependiendo de las consideraciones al momento de la estimación como la fecundidad del país o la prevalencia de los métodos de inducción de aborto. En general existen 3 modelos de aproximación:

a. Modelo de egresos hospitalarios con factores de expansión (32): Utiliza el número de egresos hospitalarios por aborto multiplicados por un factor de expansión que se calcula entrevistando a 10-15 personas que trabajan en el sistema de salud pública y que subjetivamente dan su impresión sobre la magnitud del problema. Basado en este método, los factores de expansión varían entre 4 y 9 . La mayor desventaja de este método es que no considera variables demográficas y epidemiológicas conocidas, como la fecundidad del país, las probabilidades biológicas de embarazar, el aborto espontáneo, y tampoco la natalidad o número de nacidos vivos o muertos. Por lo tanto los factores de expansión son subjetivos y no susceptibles de ser validados. Con este método se estimó para Chile en 1990 una cifra de 160.000 abortos clandestinos (34).

b. Modelo de egresos hospitalarios basados en probabilidades biológicas de aborto espontáneo (32): Este modelo utiliza valores reales disponibles en las estadísticas vitales, aplicando las probabilidades biológicas de concepción, probabilidad de aborto espontáneo antes de las 6 semanas y el aborto espontáneo clínico propiamente tal. Esto determina un "residuo" que equivaldría a una estimación de los abortos clandestinos, mediante la diferencia de los egresos por aborto y los esperados. Mediante este método se estima para Chile una cifra de 16.980 abortos clandestinos. La debilidad de este método es que requiere conocer la prevalencia del método abortivo más utilizado, y que en 
Chile debiera ser el misoprostol, cifra que no es posible de establecer.

c. Modelo de embarazos esperados (32): Este método se basa en datos objetivos de estadísticas vitales de un país y las probabilidades biológicas de los eventos reproductivos, de acuerdo a la metodología descrita por Wilcox y cols en 1995 (33). Para la estimación de los nacidos vivos esperados considera la tasa global de fecundidad, la probabilidad de concepción viable, la probabilidad de pérdida espontánea temprana y clínica, y la probabilidad de embarazos terminados en nacido vivo. La diferencia entre los nacidos esperados y los nacidos observados más los nacidos muertos, indicaría una estimación de los embarazos abortados, aplicando este método entre 1957 y 2007, se determinó una serie de tiempo que muestra una disminución desde algo más 200.000 abortos clandestinos en 1957 a 19.390 en 2007.

Estudios efectuados por el Instituto Alan Guttmacher en 1996 (34) estimó 160.000 abortos clandestinos en Chile realizados en 1990, colocando al país por encima de la mayoría de los países latinoamericanos, con una tasa estimada de aborto inducido de 45,4 aborto/1000 mujeres y 1,6 abortos/mujer; la metodología utilizada fue en base a estimaciones de los egresos hospitalarios por aborto.

Con respecto a las consideraciones utilizadas en las estimaciones, en la Tabla VI se presenta la evolución de los egresos hospitalarios por aborto en el periodo 1990-2011. No se observan cambios significativos de la tendencia de los egresos por aborto, pese a un descenso de los nacidos vivos y del total de embarazos (nacidos vivos + egresos por abortos), mientras que la relación egresos por abortos/100 embarazos y egresos por abortos/100 nacidos vivos asciende discretamente en forma significativa. Se destaca que la relación abortos/ embarazos y abortos/nacidos vivos se encuentra dentro de la frecuencia habitual esperable para el aborto espontáneo.

\section{Tabla VI}

EGRESOS HOSPITALARIOS POR ABORTO, 1990-2011

\begin{tabular}{|c|c|c|c|c|c|}
\hline AÑO & Egresos abortos* & $\begin{array}{l}\text { Nacidos vivos } \\
\text { corregidos }^{\star \star}\end{array}$ & Total embarazos & $\begin{array}{c}\text { Abortos/100 } \\
\text { nacidos vivos }\end{array}$ & $\begin{array}{l}\text { Abortos } / 100 \\
\text { embarazos }\end{array}$ \\
\hline 1990 & 36.528 & 309.220 & 345.748 & 11,8 & 10,6 \\
\hline 1991 & 36.821 & 300.740 & 337.561 & 12,2 & 10,9 \\
\hline 1992 & 34.934 & 294.218 & 329.152 & 11,9 & 10,6 \\
\hline 1993 & 32.621 & 289.419 & 322.040 & 11,3 & 10,1 \\
\hline 1994 & 30.701 & 285.228 & 315.929 & 10,8 & 9,7 \\
\hline 1995 & 28.981 & 275.760 & 304.741 & 10,5 & 9,5 \\
\hline 1996 & 30.528 & 272.163 & 302.691 & 11,2 & 10,1 \\
\hline 1997 & 31.682 & 265.493 & 297.175 & 11,9 & 10,7 \\
\hline 1998 & 30.801 & 261.802 & 292.603 & 11,8 & 10,5 \\
\hline 1999 & 30.200 & 254.096 & 284.296 & 11,9 & 10,6 \\
\hline 2000 & 30.146 & 252.155 & 282.301 & 12,0 & 10,7 \\
\hline 2001 & 34.479 & 248.651 & 283.130 & 13,9 & 12,2 \\
\hline 2002 & 34.968 & 241.027 & 275.995 & 14,5 & 12,7 \\
\hline 2003 & 33.490 & 236.223 & 269.713 & 14,2 & 12,4 \\
\hline 2004 & 33.835 & 232.588 & 266.423 & 14,5 & 12,7 \\
\hline 2005 & 33.184 & 232.092 & 265.276 & 14,3 & 12,5 \\
\hline 2006 & 33.145 & 233.104 & 266.249 & 14,2 & 12,4 \\
\hline 2007 & 32.532 & 242.054 & 274.586 & 13,4 & 11,8 \\
\hline 2008 & 33.426 & 248.366 & 281.792 & 13,5 & 11,9 \\
\hline 2009 & 33.777 & 253.574 & 287.351 & 13,3 & 11,8 \\
\hline 2010 & 31.933 & 251.199 & 283.132 & 12,7 & 11,3 \\
\hline 2011 & 30.860 & 248.879 & 279.739 & 12,4 & 11,0 \\
\hline$r=$ & $-0,1374$ & $-0,8394$ & $-0,8341$ & 0,6422 & 0,6442 \\
\hline $\mathrm{p}=$ & 0,542 & $<0,001$ & $<0,001$ & 0,001 & 0,001 \\
\hline
\end{tabular}

\footnotetext{
* Egresos Hospitalarios, DEIS. ** INE. Estadísticas vitales 2011 (22).
} 
En 2012, última estadística entregada por el MINSAL, muestra un total de egresos por aborto de 30.434 , de ellos $130(0,43 \%)$ correspondieron a adolescentes de 10-14 años y $3.070(10,1 \%)$ a adolescentes de 15-19 años (35). Es evidente que el aborto clandestino existía y existe en Chile, pero es difícil entender una estimación de 160.000 abortos clandestinos (1990), época en la cual ese tipo de aborto se realizaba con métodos muy cruentos y posiblemente la mayoría de ellos debían recurrir finalmente a las Maternidades para tratamiento por abortos incompletos, infección o hemorragias.

En diciembre de 2013, Molina y cols (38), en base a los egresos y tipos de causas del aborto, estimaron que con la incorporación del misoprostol los egresos por los supuestos abortos clandestinos que requirieron hospitalización, deberían multiplicarse por 6, lo que daría una cifra de abortos ilegales en Chile de 109.200 (72.800-145.600) abortos anuales en la actualidad.

Se ha comunicado que las leyes restrictivas al aborto aumentan el riesgo de aborto inseguro y la mortalidad por aborto, y que la descriminalización rápidamente reduce la mortalidad materna por aborto y no aumenta la tasa de abortos (39). En Chile, posterior al cambio de la ley en 1989, la mortalidad por aborto continuó descendiendo desde 10,8/100.000 nacidos vivos a 0,39/100.000 nacidos vivos en 2009 , equivalente a un descenso de $96,3 \%$ (40), por lo que en Chile la ilegalidad del aborto no se ha traducido en un aumento de la mortalidad materna por aborto, lo que permite inferir que el aborto inducido también habría disminuido.

\section{CONCLUSIONES}

- Chile en la década de los 60 tenía una de las tasas de mortalidad materna por aborto inducido más altas de América Latina.

- La introducción de los métodos de planificación familiar en Chile en la década de los 60 del siglo XX permitió a la mujer chilena el control de su fecundidad y como consecuencia la reducción significativa de la mortalidad materna general y por aborto inducido.

- En la actualidad podemos afirmar que en Chile la muerte materna por aborto deja de ser el problema de salud pública que era antes de 1965.

- En Chile la significativa disminución de la mortalidad materna general y por aborto refleja el desarrollo social, educacional y sanitario del país.

- El cambio de legislación sobre la ilegalidad del aborto en todas sus formas e indicaciones realizada en 1989, no ha producido un aumento de la mortalidad materna por aborto.

- Se aprecia una sobrestimación de la magnitud del aborto ilegal en Chile, dado por estimaciones con importantes limitaciones metodológicas.
- Chile ha logrado descender la mortalidad materna por aborto sin recurrir a la legalización del aborto respetando al embrión humano desde la concepción.

\section{REFERENCIAS}

1. Astete C, Beca JP, Lecaros A. Propuesta de un glosario para la discusión del aborto. Rev Med Chile 2014;142(11):1149-51.

2. PROSEGO. Protocolos asistenciales en obstetricia. Aborto espontáneo. En: Sociedad Española de Obstetricia y Ginecología, editor. Madrid: Sociedad Española de Obstetricia y Ginecología, 2010. Disponible en: http://apps.elsevier.es/watermark/ctl_ servlet?_f=10\&pident_articulo=90003117\&pident_ usuario $=0 \&$ pcontactid $=\&$ pident_revista $=151 \&$ ty $=3 \& a$ ccion $=$ L\&origen $=$ zonadelectura $\& w e b=w w w$.elsevier. es\&lan=es\&fichero $=151 \mathrm{v} 54 \mathrm{n} 04 \mathrm{a} 90003117 \mathrm{pdf001}$. pdf.

3. World Health Organisation. International Statistical Classification of Diseases and Related Health Problems. 10th Revision. En: WHO, editor, 2010. Disponible en: http://www.who.int/classifications/icd/en/.

4. Raju TN, Mercer BM, Burchfield DJ, Joseph GF. Periviable birth: executive summary of a joint workshop by the Eunice Kennedy Shriver National Institute of Child Health and Human Development, Society for Maternal-Fetal Medicine, American Academy of Pediatrics, and American College of Obstetricians and Gynecologists. Obstet Gynecol 2014;123(5):1083-96.

5. Silva S. Aborto. En: Pérez Sánchez A, Donoso Siña E, editores. Obstetricia. 4a Edición. Editorial Maditerráneo. 2011. pp 530-561.

6. Goddijn M, Leschot NJ. Genetic aspects of miscarriage. Baillieres Best Pract Res Clin Obstet Gynaecol 2000;14(5):855-65.

7. van den Berg MM, van Maarle MC, van Wely M, Goddijn M. Genetics of early miscarriage. Biochim Biophys Acta 2012;1822(12):1951-9.

8. OMS. Aborto sin riesgo. Guía técnica y de políticas para sistemas de salud. Segunda Edición. 2012. Disponible en: www.who.int/iris/bitstre am/10665/77079/1/9789243548432_spa.pdf.

9. Ventura SJ, Curtin SC, Abma JC, Henshaw SK. Estimated pregnancy rates and rates of pregnancy outcomes for the United States, 1990-2008. Natl Vital Stat Rep 2012;60(7):1-21.

10. Bartlett LA, Berg CJ, Shulman HB, Zane SB, Green $\mathrm{CA}$, Whitehead S, et al. Risk factors for legal induced abortion-related mortality in the United States. Obstet Gynecol 2004;103(4):729-37.

11. Zane S, Creanga AA, Berg CJ, Pazol K, Suchdev $\mathrm{DB}$, Jamieson DJ, et al. Abortion-Related Mortality in the United States: 1998-2010. Obstet Gynecol 2015;126(2):258-65.

12. Berer M. Making abortions safe: a matter of good public health policy and practice. Bull World Health Organ 2000;78(5):580-92.

13. Lamper-Grassi MP. Aborto en Chile. Evolución histórica del marco normativo. Asesorias Parlamentarias - Análisis Descriptivo y Evaluativo. Biblioteca del Congreso Nacional de Chile, 2015. Disponible en: http://www.bcn. 
$\mathrm{cl} /$ Buscadorlntegral/buscar?contenido=Asesorias $\% 20$ Parlamentarias\&texto=codigo\%20del\%20trabajo\&con ector=AND\&orden=DRETITLE:alphabetical+Relevanc e+Date\&res_x_pag=10.

14. S.E. la Presidenta de la República. Mensaje № 1230362, 31 enero 2015, Boletín $N^{\circ}$ 9895-11. Disponible en: https://www.camara.cl/pley/pley_detalle.aspx?pr $\mathrm{mID}=10315$ \&prmBoletin=9895-11.

15. Instituto Nacional de Estadísticas. Estadísticas Vitales. Anuario 2013. Disponible en: http://www.ine.cl/ canales/menu/publicaciones/calendario_de_publicaciones/pdf/completa_vitales_2013.pdf.

16. Donoso E. ¿Unsafe abortion en Chile? Rev Chil Obstet Ginecol 2008;73(6):359-61.

17. Armijo R, Requena M. Aspectos epidemiológicos del aborto. Bol Oficina Sanit Panam 1967;63(5):391-401.

18. Muñoz W, Cuevas G, Becker A. Mortalidad materna: revisión de la casuística de 30 años. Rev Chil Obstet Ginecol 1980;45(3):159-69.

19. Armijo R, Monreal T. El problema del aborto provocado en Chile. Bol Oficina Sanit Panam 1966;60(1):3945.

20. Molina R, López J, Orellana M, Orlandi E, Alarcón J. Características del aborto en Chile. 1978. Cuadernos Médico Sociales. Disponible en: http://cms.colegiomedico.cl/Magazine/1978/19/1/19_1_5.pdf.

21. Szot J, Moreno C. Mortalidad por aborto en Chile: análisis epidemiológico 1985-2000. Rev Chil Obstet Ginecol 2003;68(4):309-14.

22. Instituto Nacional de Estadísticas. Anuarios de Estadísticas Vitales 1990-2011. Disponible en: http://www. ine.cl/canales/chile_estadistico/familias/demograficas_vitales.php.

23. MINSAL. Subsecretaría de Salud Pública. Orientaciones técnicas. Para la atención integral de mujeres que presentan un aborto y otras pérdidas reproductivas. 2011. Disponible en: http://web.minsal.cl/portal/url/ item/b53faf5d1d527a52e04001011e010ef5.pdf.

24. Donoso E, Carvajal JA. El cambio del perfil epidemiológico de la mortalidad materna en Chile dificultará el cumplimiento del $5^{\circ}$ objetivo del Milenio. Rev Med Chile 2012;140(10):1253-62.

25. Koch E, Thorp J, Bravo M, Gatica S, Romero CX, Aguilera $\mathrm{H}$, et al. Women's education level, maternal health facilities, abortion legislation and maternal deaths: a natural experiment in Chile from 1957 to 2007. PLoS One 2012;7(5):e36613.

26. Encuesta de Calidad de Vida y Salud (ENCAVI 2000). Informe Planificación Familiar. Módulo II. Hallado en: http://www.ine.cl/canales/chile_estadistico/calidad_ de_vida_y_salud/calidadvida/infplanfam.pdf.

27. Conde-Agudelo A, Belizan JM, Lammers C. Maternalperinatal morbidity and mortality associated with adolescent pregnancy in Latin America: Cross-sectional study. Am J Obstet Gynecol 2005;192(2):342-9.
28. INJUV. 6ta Encuesta Nacional de la Juventud: Instituto Nacional de la Juventud, Gobierno de Chile, 2010. Disponible en: http://www.injuv.gob.cl/portal/wp-content/files_mf/sextaencuestanacionaldejuventud.pdf.

29. INJUV. $7 \mathrm{ma}$ Encuesta Nacional de Juventud 2012: Ministerio de Desarrollo Social. Gobierno de Chile., 2012. Disponible en: http://www.injuv.gob.cl/portal/ wp-content/files_mf/septimaencuestanacionaljuventud2.pdf.

30. MINSAL. Situación actual del embarazo adolescente en Chile: Ministerio de Salud de Chile, 2013. Disponible en: http://web.minsal.cl/portal/url/item/c908a2010f2e7dafe040010164010db3.pdf.

31. Stover J, Ross J. How increased contraceptive use has reduced maternal mortality. Matern Child Health $\mathrm{J}$ 2010;14(5):687-95.

32. Koch E. Presentación a la Comisión de Salud del Senado de Chile. Despenalización de la interrupción del embarazo por razones médicas. Boletin 7373-07, 2011. Disponible en: http://www.bioeticaweb.com/ wp-content/uploads/2011/08/aborto-y-mortalidad-materna-en-chile-presentacion-del-dr-koch-ante-senado-2011.pdf.

33. Wilcox AJ, Weinberg CR, Baird DD. Timing of sexual intercourse in relation to ovulation. Effects on the probability of conception, survival of the pregnancy, and sex of the baby. N Engl J Med 1995;333(23):1517-21.

34. The Alan Guttmacher Institute. Temas en breve. Panorama general del aborto clandestino en América Latina, 1996. Disponible en: http://www.guttmacher. org/pubs/ib12sp.pdf.

35. Departamento de Estadísticas e Información de Salud. Egresos Hospitalarios. 2012. Disponible en: http://intradeis.minsal.cl/egresoshospitalarios/menu_ publica_nueva/menu_publica_nueva.htm.

36. Prada E, Singh S, Remez L, Villarreal. Unintended pregnancy and induced abortion in Colombia. Causes and Consequences. 2011. Disponible en: http://www. guttmacher.org/pubs/Embarazo-no-deseado-Colombia.pdf.

37. Koch E, Bravo M, Gatica S, Stecher JF, Aracena P, Valenzuela $S$, et al. Sobreestimación de la ocurrencia de abortos inducidos en Colombia y otros países latinoamericanos. Ginecol Obstet Mex 2012;80(5):36072.

38. Molina-Cartes R, Molina T, Carrasco X, Eguiguren $\mathrm{P}$. Profile of abortion in Chile, with extremely restrictive law. Open J Obstet Gynecol 2013;3:732-8. Disponible en: http://file.scirp.org/pdf/ OJOG_2013122409440632.pdf.

39. Faundes A, Shah IH. Evidence supporting broader access to safe legal abortion. Int J Gynaecol Obstet 2015;131 Suppl 1: S56-9.

40. Koch E. Epidemiología del aborto y su prevención en Chile. Rev Chil Obstet Ginecol 2014;79(5):351-60. 